\title{
28. DATA REPORT: ABUNDANCE PATTERNS OF PLANKTONIC FORAMINIFERS: SITE 904, LEG 150'
}

\author{
Scott W. Snyder ${ }^{2}$
}

\begin{abstract}
Calculation of the number of planktonic foraminifers per gram (dry weight) of sediment reveals dramatic changes in abundance within Eocene to Miocene sediments at Site 904 . The largest scale changes (264 samples analyzed) parallel regional trends in paleoclimate and terrigenous sediment input. Abundance changes of shorter duration, some equivalent in scale to long term fluctuations, reflect the complex interaction of global sea-level change, diagenesis, paleoproductivity, and sediment accumulation rate. Data presented here are intended to support subsequent studies that may unravel some of this complexity. My purpose is to help identify the dominant processes associated with abundance changes of the planktonic foraminifers.
\end{abstract}

\section{INTRODUCTION}

The abundance of planktonic microfossils is fundamental to the definition and interpretation of selected systems tracts in models of sequence stratigraphy (Van Wagoner et al., 1988). Understanding these systems tracts and their relationships to bounding unconformities is, in turn, prerequisite to correlating sequences with models of global eustatic sea-level fluctuation (Haq et al., 1987). Of particular importance are condensed sections, which are recognized, in part, by high concentrations of planktonic microfossils (Loutit et al., 1988). A primary objective of Leg 150 was to investigate the timing and magnitude of past global sea-level changes (Miller et al., 1994). Site 904, which has the most abundant and continuous record of planktonic foraminifers among the sites that were drilled (Snyder et al., this volume), offers a unique opportunity to explore the significance of abundance trends in Eocene through Miocene sediments.

One might argue that total carbonate content, which can be determined more quickly and easily, should mirror the abundance trends of planktonic microfossils. In the Eocene section, where planktonic foraminifers are abundant, typically two or three orders of magnitude more abundant than in most of the Miocene section, that might be true. However, where planktonic foraminifers are sparse, other faunal elements may represent as much or more of the total carbonate content. For example, some samples lacking planktonic foraminifers contain benthic foraminifers. In other samples, where planktonic specimens are rare, benthic foraminifers are the dominant calcareous microfossils. Ostracodes, echinoid spines, and fragments of larger shells, although rare and sporadic in occurrence, collectively account for most of the carbonate in some samples. Hence, total carbonate content in many Oligocene and Miocene samples would not accurately reflect abundance of planktonic microfossils. The most reliable way to estimate such abundance is through a census of the planktonic microfossils, in this case foraminifers.

\section{METHODS}

Preparation began by oven-drying bulk sediment samples at $45^{\circ} \mathrm{C}$ for $24 \mathrm{hr}$. Each dried sample was then weighed to the nearest milli-

'Mountain, G.S., Miller, K.G., Blum, P., Poag, C.W., and Twichell, D.C. (Eds.), 1996. Proc. ODP, Sci. Results, 150: College Station, TX (Ocean Drilling Program).

${ }^{2}$ Department of Geology, East Carolina University, Greenville, NC 27858, U.S.A glsnyder@ecuvm.cis.ecu.edu gram on a Sartorius B-120-S balance, transferred to a $1000-\mathrm{mL}$ beaker, and immersed in approximately $250 \mathrm{~mL}$ of a $3 \% \mathrm{H}_{2} \mathrm{O}_{2}$ solution. After soaking for $24 \mathrm{hr}$ at $45^{\circ} \mathrm{C}$, the sample- $\mathrm{H}_{2} \mathrm{O}_{2}$ mixture was heated for $15 \mathrm{~min}$ and then gently washed over a \#230 U.S. Standard Sieve (63- $\mu \mathrm{m}$ mesh).

If the clay-silt fraction was not entirely disaggregated, the soaking, heating, and sieving procedures were repeated (a maximum of three times). Many samples, particularly those from the Neogene section, required only a single washing; others, largely from the middle Eocene section, required three washes. Preservation of fragile species and delicate ornamentation in foraminifer-rich samples indicates that sample preparation did not preferentially destroy specimens or bias counts. The final wash utilized a \#18 U.S. Standard Sieve (1-mm mesh) to separate coarser material, if present, from the sand fraction concentrated on the \#230 sieve. Samples were then oven-dried at $45^{\circ} \mathrm{C}$ and both the sand fraction and coarse fraction, when present, were weighed and transferred to labeled vials.

Samples in which silts and clays were completely dispersed generally produced no coarse fraction. Rarely, the coarse fraction included large benthic foraminifers, otoliths(?), $\mathrm{CaCO}_{3}$ fragments of larger shells, or quartz grains. In some samples, $90 \%$ of which came from the middle Eocene porcellanitic chalks below Sample 150-904A$53 \mathrm{X}-1,39-41 \mathrm{~cm}$, at 490.6 meters below seafloor (mbsf), the coarse fraction consisted of aggregate grains composed of silts and clays, which persisted even after three cycles of processing. In such cases, the weight of these aggregate grains was subtracted from the weight of the initial bulk sediment sample in order to calculate the weight of sample from which foraminifers were actually recovered. For example, bulk sediment Sample 150-904A-58X-6, 39-41 cm, weighed $15.981 \mathrm{~g}$. After three washings, the sand fraction weighed $0.604 \mathrm{~g}$ and the "coarse fraction" (composed entirely of nondisaggregated mud) weighed $3.210 \mathrm{~g}$, meaning that foraminiferal counts had to be computed relative to the $12.771 \mathrm{~g}$ actually broken down to yield the specimens. This approach also allowed calculation of the percentage of mud in each sample. Percentages of mud and sand for each sample are listed in Table 1 and plotted in Figures 1 and 2, respectively.

For foraminifer-rich samples, the washed sand fraction retained on the $63-\mu \mathrm{m}$-mesh sieve was divided into aliquots using a microsplitter. Dry-sieving isolated the $>150-\mu \mathrm{m}$ fraction of the sample split, from which all planktonic foraminifers were then counted. Of course, total abundances vary markedly depending upon the size fraction examined. Various authors have recommended the use of specific size fractions for analysis of foraminifers. Schroeder et al. (1987) recommended using the $>63-\mu \mathrm{m}$ fraction, whereas $\mathrm{Lu}$ and 
Table 1. Number of planktonic foraminifers per gram of sediment and percentages of mud and sand in Hole 904A (calculations described in text).

\begin{tabular}{|c|c|c|c|c|}
\hline $\begin{array}{l}\text { Core, section, } \\
\text { interval }(\mathrm{cm})\end{array}$ & $\begin{array}{l}\text { Depth } \\
\text { (mbsf) }\end{array}$ & $\begin{array}{c}\text { Planktonic } \\
\text { foraminifers/gram }\end{array}$ & $\begin{array}{l}\text { Sand } \\
(\%)\end{array}$ & $\begin{array}{l}\text { Mud } \\
(\%)\end{array}$ \\
\hline \multicolumn{5}{|l|}{$150-904 \mathrm{~A}-$} \\
\hline $17 \mathrm{H}-\mathrm{I}, 39-41$ & 149.39 & 0.0 & 9.2 & 90.8 \\
\hline $17 \mathrm{H}-2,39-41$ & 150.89 & 0.0 & 13.5 & 86.5 \\
\hline $17 \mathrm{H}-3,39-41$ & 152,39 & 0.0 & 21.5 & 78.5 \\
\hline $17 \mathrm{H}-4,39-41$ & 153.89 & 0.0 & 26.5 & 73.5 \\
\hline $17 \mathrm{H}-5,39-41$ & 155.39 & 1.0 & 28.4 & 71.6 \\
\hline $17 \mathrm{H}-6,39-41$ & 156.89 & 0.0 & 41.2 & 58.8 \\
\hline $17 \mathrm{H}-7,39-41$ & 158.39 & 0.0 & 4.6 & 95.4 \\
\hline $18 \mathrm{H}-1,39-41$ & 158.89 & 0.0 & 4.2 & 95.8 \\
\hline $18 \mathrm{H}-2,39-41$ & 160.39 & 0.0 & 8.6 & 91.4 \\
\hline $18 \mathrm{H}-3,38-40$ & 161.88 & 0.0 & 19.9 & 80.1 \\
\hline $18 \mathrm{H}-4,39-41$ & 163.39 & 0.0 & 5.7 & 94.3 \\
\hline $18 \mathrm{H}-5,39-41$ & 164.89 & 0.0 & 91.7 & 8.3 \\
\hline $18 \mathrm{H}-6,39-41$ & 166.39 & 0.0 & 86.0 & 14.0 \\
\hline $18 \mathrm{H}-7,42-44$ & 167.42 & 0.0 & 88.1 & 11.9 \\
\hline $19 \mathrm{H}-1,39-41$ & 168.39 & 0.0 & 0.9 & 99.1 \\
\hline $19 \mathrm{H}-2,39-41$ & 169.89 & 0.0 & 0.8 & 99.2 \\
\hline $19 \mathrm{H}-3,39-41$ & 171.39 & 0.3 & 0.7 & 99.3 \\
\hline $19 \mathrm{H}-4,39-41$ & 172.89 & 0.0 & 0.6 & 99.4 \\
\hline $19 \mathrm{H}-5,39-41$ & 174.39 & 0.0 & 1.5 & 98.5 \\
\hline $20 \mathrm{H}-1,40-42$ & 177.90 & 0.0 & 2.5 & 97.5 \\
\hline $20 \mathrm{H}-2,40-42$ & 179.40 & 1.1 & 13.1 & 86.9 \\
\hline $20 \mathrm{H}-3,40-42$ & 180.90 & 0.0 & 1.5 & 98.5 \\
\hline $20 \mathrm{H}-4,40-42$ & 182.40 & 4.2 & 8.4 & 91.6 \\
\hline $21 \mathrm{H}-1,40-42$ & 187.40 & 0.0 & 1.5 & 98.5 \\
\hline $21 \mathrm{H}-2,40-42$ & 188.90 & 0.7 & 2.4 & 97.6 \\
\hline $21 \mathrm{H}-3,40-42$ & 190.40 & 0.0 & 1.4 & 98.6 \\
\hline $21 \mathrm{H}-4,40-42$ & 191.90 & 29.9 & 1.3 & 98.7 \\
\hline $22 \mathrm{H}-1,40-42$ & 196.90 & 0.0 & 5.2 & 94.8 \\
\hline $22 \mathrm{H}-2,40-42$ & 198.40 & 0.0 & 1.6 & 98.4 \\
\hline $22 \mathrm{H}-3,40-42$ & 199.90 & 0.0 & 0.0 & 100.0 \\
\hline $22 \mathrm{H}-4,40-42$ & 201.40 & 1.2 & 1.1 & 98.9 \\
\hline $22 \mathrm{H}-5,40-42$ & 202.40 & 0.0 & 1.3 & 98.7 \\
\hline $23 \mathrm{H}-1,40-42$ & 203.40 & 0.0 & 0.7 & 99.3 \\
\hline $23 \mathrm{H}-2,40-42$ & 204,90 & 0.1 & 1.5 & 98.5 \\
\hline $23 \mathrm{H}-3,40-42$ & 206.40 & 1.0 & 2.1 & 97.9 \\
\hline $23 \mathrm{H}-4,40-42$ & 207.90 & 4.8 & 2.9 & 97.1 \\
\hline $23 \mathrm{H}-5,40-42$ & 209.42 & 0.0 & 1.5 & 98.5 \\
\hline $24 \mathrm{H}-1,40-42$ & 212.90 & 125.4 & 8.9 & 91.1 \\
\hline $24 \mathrm{H}-2,40-42$ & 214.40 & 39.4 & 3.5 & 96.5 \\
\hline $24 \mathrm{H}-4,40-42$ & 217.40 & 3.9 & 37.4 & 62.6 \\
\hline $24 \mathrm{H}-5,40-42$ & 218.90 & 2.8 & 23.2 & 76.8 \\
\hline $24 \mathrm{H}-6,42-44$ & 220.42 & 0.0 & 1.8 & 98.2 \\
\hline $25 X-1,68-70$ & 222.68 & 0.0 & 4.4 & 95.6 \\
\hline $25 \mathrm{X}-2,40-42$ & 223.90 & 0.0 & 4.0 & 96.0 \\
\hline $25 \times-3,40-42$ & 225.40 & 0.0 & 31.7 & 68.3 \\
\hline $25 X-4,40-42$ & 226.90 & 0.0 & 27.6 & 72.4 \\
\hline $25 X-5,40-42$ & 228.40 & 0.0 & 39.8 & 60.2 \\
\hline $25 \mathrm{X}-6,40-42$ & 229.90 & 0.0 & 47.7 & 52.3 \\
\hline $26 \mathrm{X}-1,40-42$ & 231.70 & 0.0 & 22.6 & 77.4 \\
\hline $26 \mathrm{X}-2,40-42$ & 233.20 & 0.0 & 14.0 & 86.0 \\
\hline $26 \times-3,40-42$ & 234.70 & 0.0 & 32.2 & 67.8 \\
\hline $26 X-4,40-42$ & 236.20 & 0.0 & 34.4 & 65.6 \\
\hline $26 X-5,40-42$ & 237.70 & 0.0 & 22.2 & 77.8 \\
\hline $26 \times-6,40-42$ & 239.20 & 0.0 & 19.5 & 80.5 \\
\hline $26 \mathrm{X}-7,40-42$ & 240.70 & 3.3 & 18.5 & 81.5 \\
\hline $27 X-1,40-42$ & 241.30 & 14.3 & 37.4 & 62.6 \\
\hline $27 \mathrm{X}-2,40-42$ & 242.80 & 33.6 & 36.2 & 63.8 \\
\hline $27 X-3,40-42$ & 244.30 & 12.4 & 42.3 & 57.7 \\
\hline $27 X-4,41-43$ & 245.81 & 35.6 & 22.4 & 77.6 \\
\hline $27 X-5,40-42$ & 247.30 & 26.1 & 42.2 & 57.8 \\
\hline $27 X-6,40-42$ & 248.80 & 29.5 & 17.2 & 82.8 \\
\hline $28 X-1,40-42$ & 250.70 & 17.7 & 36.9 & 63.1 \\
\hline $28 X-2,40-42$ & 252.20 & 167.3 & 26.2 & 73.8 \\
\hline $28 X-3,40-42$ & 253.70 & 0.0 & 42.2 & 57.8 \\
\hline $28 X-4,40-42$ & 255.20 & 5.9 & 38.3 & 61.7 \\
\hline $28 X-5,40-42$ & 256.75 & 28.0 & 44.9 & 55,1 \\
\hline $29 \mathrm{X}-1,40-42$ & 260.50 & 164.6 & 57.2 & 42.8 \\
\hline $29 X-2,40-42$ & 262.00 & 51.6 & 29.7 & 70.3 \\
\hline $29 \mathrm{X}-3,40-42$ & 263.50 & 3.7 & 27.8 & 72.2 \\
\hline $29 \times-4,40-42$ & 265.00 & 43.7 & 34.4 & 65.6 \\
\hline $29 X-5,40-42$ & 266.50 & 7.1 & 5.8 & 94.2 \\
\hline $29 X-6,40-42$ & 268.00 & 0.0 & 20.9 & 79.1 \\
\hline $29 X-7,75-76$ & 268.35 & 155.1 & 20.2 & 79.8 \\
\hline $30 X-2,36-38$ & 271.66 & 193.0 & 36.1 & 63.9 \\
\hline $30 X-3,40-42$ & 273.20 & 0.0 & 24.8 & 75.2 \\
\hline $30 \times-4,40-42$ & 274.70 & 3.1 & 34.4 & 65.6 \\
\hline $30 X-5,40-42$ & 276.20 & 0.0 & 32.4 & 67.6 \\
\hline $30 X-6,40-42$ & 277.70 & 0.0 & 31.8 & 60.2 \\
\hline $30 \times-7,40-42$ & 279.20 & 0.0 & 37.7 & 62.3 \\
\hline $31 X-1,40-42$ & 279.90 & 0.0 & 36.9 & 63.1 \\
\hline $31 X-2,40-42$ & 281.40 & 4.4 & 26.4 & 73.6 \\
\hline $31 X-3,40-42$ & 282.90 & 0.0 & 30.3 & 69.7 \\
\hline $31 X-4,40-42$ & 284.40 & 0.0 & 71.9 & 28.1 \\
\hline $31 \times-5,40-42$ & 285.90 & 0.0 & 38.9 & 61.1 \\
\hline $31 X-6,40-42$ & 287.40 & 0.0 & 31.7 & 68.3 \\
\hline $31 \times-7,40-42$ & 288.90 & 0.0 & 9.5 & 90.5 \\
\hline
\end{tabular}

\begin{tabular}{|c|c|c|c|c|}
\hline $\begin{array}{l}\text { Core, section, } \\
\text { interval }(\mathrm{cm})\end{array}$ & $\begin{array}{l}\text { Depth } \\
\text { (mbsf) }\end{array}$ & $\begin{array}{c}\text { Planktonic } \\
\text { foraminifers/gram }\end{array}$ & $\begin{array}{l}\text { Sand } \\
(\%)\end{array}$ & $\begin{array}{l}\text { Mud } \\
(\%)\end{array}$ \\
\hline $32 X-1,40-42$ & 289.50 & 6.4 & 17.0 & 83.0 \\
\hline $32 X-2,40-42$ & 291.00 & 4.3 & 19.5 & 80.5 \\
\hline $32 \times-3,40-42$ & 292.50 & 11.6 & 25.2 & 74.8 \\
\hline $32 \times-4,40-42$ & 294.00 & 8.6 & 31.2 & 68.8 \\
\hline $32 X-5,40-42$ & 295.50 & 6.9 & 31.6 & 68.4 \\
\hline $32 X-6,40-42$ & 297.00 & 3001.2 & 18.6 & 81.4 \\
\hline $32 \times-7,40-42$ & 298.50 & 5982.6 & 17.9 & 82.1 \\
\hline $33 \mathrm{X}-1,40-42$ & 299.20 & 3160.7 & 18.5 & 81.5 \\
\hline $33 \mathrm{X}-2,40-42$ & 300.70 & 2038.5 & 7.2 & 92.8 \\
\hline $33 X-3,40-42$ & 302.20 & 170.3 & 19.8 & 80.2 \\
\hline $33 \times-4,40-42$ & 303.70 & 2638.9 & 18.9 & 81.1 \\
\hline $33 X-5,40-42$ & 305.20 & 52.6 & 18.2 & 81.8 \\
\hline $33 \mathrm{X}-6,40-42$ & 306.70 & 388.7 & 21.8 & 78.2 \\
\hline $33 X-7,40-42$ & 308.20 & 7780.7 & 23.2 & 76.8 \\
\hline $34 X-1,40-42$ & 308.79 & 6047.4 & 20.3 & 79.7 \\
\hline $34 X-2,40-42$ & 310.30 & 303.5 & 18.6 & 81.4 \\
\hline $34 \mathrm{X}-3,40-42$ & 311.80 & 359.2 & 36.4 & 63.6 \\
\hline $34 X-4,40-42$ & 313.30 & 1018.2 & 25.4 & 74.6 \\
\hline $34 X-5,40-42$ & 314.80 & 3093.0 & 24.1 & 75.9 \\
\hline $34 \mathrm{X}-6,40-42$ & 316.30 & 66.4 & 23.8 & 76.2 \\
\hline $34 X-7,40-42$ & 317.80 & 42.6 & 36.2 & 63.8 \\
\hline $35 \mathrm{X}-1,39-41$ & 318.19 & 43.7 & 30.9 & 69.1 \\
\hline $35 \times-2,39-41$ & 319.69 & 95.3 & 20.1 & 79.9 \\
\hline $35 \mathrm{X}-3,39-41$ & 321.19 & 145.6 & 15.6 & 84.4 \\
\hline $35 \times-4,39-41$ & 322.69 & 2696.2 & 17.3 & 82.7 \\
\hline $35 X-5,39-41$ & 324.19 & 370.2 & 12.5 & 87.5 \\
\hline $35 \mathrm{X}-6,39-41$ & 325.69 & 132.8 & 21.6 & 78.4 \\
\hline $35 X-7,39-41$ & 327.19 & 90.7 & 14.0 & 86.0 \\
\hline $36 \mathrm{X}-1,39-41$ & 327.59 & 22.7 & 15.8 & 84.2 \\
\hline $36 \mathrm{X}-2,39-41$ & 329.09 & 51.7 & 25.2 & 74.8 \\
\hline $36 \mathrm{X}-3,39-41$ & 330.59 & 20.1 & 34.4 & 65.6 \\
\hline $36 \times-4,39-41$ & 332.09 & 9.4 & 35.3 & 64.7 \\
\hline $36 \times-5,39-41$ & 333.59 & 28.1 & 33.2 & 66.8 \\
\hline $36 \times-6,39-41$ & 335.09 & 28.9 & 27.6 & 72.4 \\
\hline $36 \times-7,39-41$ & 336.09 & 64.7 & 22.2 & 77.8 \\
\hline $37 \mathrm{X}-2,36-38$ & 338.36 & 35.0 & 52.1 & 47.9 \\
\hline $37 \mathrm{X}-3,39-41$ & 339.89 & 14.1 & 56.6 & 43.4 \\
\hline $37 X-4,44-46$ & 341.44 & 174.7 & 32.2 & 67.8 \\
\hline $37 X-5,39-41$ & 342.89 & 687.7 & 38.4 & 61.6 \\
\hline $37 \mathrm{X}-6,39-41$ & 344.39 & 336.8 & 26.5 & 73.5 \\
\hline $38 \mathrm{X}-1,39-41$ & 346.19 & 304.7 & 17.2 & 82.8 \\
\hline $38 \mathrm{X}-2,41-43$ & 347.71 & 140.1 & 16.0 & 84.0 \\
\hline $38 \times-3,39-41$ & 349.19 & 267.2 & 11.6 & 88.4 \\
\hline $38 \times-4,39-41$ & 350.69 & 65.1 & 5.8 & 94.2 \\
\hline $38 \mathrm{X}-5,39-41$ & 352.19 & 194.6 & 8.1 & 91.9 \\
\hline $38 \mathrm{X}-6,39-41$ & 353.69 & 828.0 & 8.3 & 91.7 \\
\hline $38 X-7,39-41$ & 354.69 & 872.0 & 10.6 & 89.4 \\
\hline $39 \mathrm{X}-1,39-41$ & 355.89 & 998.6 & 6.8 & 93.2 \\
\hline $39 X-2,39-41$ & 357.39 & 327.4 & 11.0 & 89.0 \\
\hline $39 \times-3,39-41$ & 358.89 & 512.9 & 7.0 & 93.0 \\
\hline $39 X-4,38-40$ & 360.38 & 421.0 & 4.2 & 95.8 \\
\hline $39 X-5,40-42$ & 361.90 & 284.6 & 5.9 & 94.1 \\
\hline $39 X-6,40-42$ & 363.40 & 454.4 & 9.9 & 90.1 \\
\hline $39 \times-7,40-42$ & 364.90 & 272.9 & 3.9 & 96.1 \\
\hline $40 X-1,40-41$ & 365.50 & 848.8 & 7.0 & 93.0 \\
\hline $40 \mathrm{X}-2,40-41$ & 367.00 & 507.8 & 11.6 & 88.4 \\
\hline $40 X-3,40-41$ & 368.50 & 245.8 & 7.7 & 92.3 \\
\hline $40 \times-4,40-41$ & 370.00 & 287.6 & 3.8 & 96.2 \\
\hline $40 X-5,40-41$ & 371.50 & 246.3 & 6.4 & 93.6 \\
\hline $40 X-6,40-41$ & 373.00 & 642.0 & 4.5 & 95.5 \\
\hline $41 X-1,40-42$ & 375.20 & 365.9 & 5.5 & 94.5 \\
\hline $41 X-2,40-42$ & 376.70 & 295.6 & 4.6 & 95.4 \\
\hline $41 \times-3,40-42$ & 378.20 & 270.8 & 3.7 & 96.3 \\
\hline $41 \times-4,40-42$ & 379.70 & 185.4 & 2.9 & 97.1 \\
\hline $41 X-5,40-42$ & 381.20 & 637.8 & 5.8 & 94.2 \\
\hline $41 \times-6,40-42$ & 382.70 & 653.4 & 7.4 & 92.6 \\
\hline $41 X-7,40-42$ & 384.20 & 201.3 & 3.8 & 96.2 \\
\hline $42 X-1,40-42$ & 384.80 & 419.0 & 7.3 & 92.7 \\
\hline $42 X-2,40-42$ & 386.30 & 193.6 & 7.3 & 92.7 \\
\hline $42 X-3,40-42$ & 387.80 & 459.8 & 5.0 & 95.0 \\
\hline $42 \times-4,40-42$ & 389.30 & 372.6 & 4.0 & 96.0 \\
\hline $42 X-5,40-42$ & 390.80 & 111.2 & 5.2 & 94.8 \\
\hline $42 \times-6,40-42$ & 392.30 & 246.0 & 3.1 & 96.9 \\
\hline $43 X-1,40-42$ & 394.50 & 426.3 & 3.7 & 96.3 \\
\hline $43 \mathrm{X}-2,40-42$ & 396.00 & 229.6 & 3.6 & 96.4 \\
\hline $43 \times-3,37-39$ & 397.47 & 414.8 & 4.0 & 96.0 \\
\hline $43 \times-4,40-42$ & 399.00 & 539.8 & 3.2 & 96.8 \\
\hline $43 \times-5,37-39$ & 400.47 & 476.8 & 15.6 & 84.4 \\
\hline $43 \times-6,40-42$ & 402.00 & 153.4 & 3.7 & 96.3 \\
\hline $44 X-1,40-42$ & 404.20 & 180.2 & 11.7 & 88.3 \\
\hline $44 X-2,40-42$ & 405.70 & 196.3 & 3.4 & 96.6 \\
\hline $44 X-3,39-41$ & 407.19 & 199.2 & 1.5 & 98.5 \\
\hline $44 X-4,39-41$ & 408.69 & 222.6 & 2.9 & 97.1 \\
\hline $44 X-5,39-41$ & 410.19 & 158.7 & 2.9 & 97.1 \\
\hline $44 X-6,40-42$ & 411.70 & 257.3 & 3.7 & 96.3 \\
\hline $44 X-7,41-43$ & 413.21 & 445.2 & 3.0 & 97.0 \\
\hline $45 X-1,40-42$ & 413.80 & 147.7 & 2.1 & 97.9 \\
\hline
\end{tabular}


Table 1 (continued).

\begin{tabular}{lrrrr}
\hline Core, section, & $\begin{array}{c}\text { Depth } \\
\text { interval (cm) }\end{array}$ & $\begin{array}{c}\text { Planktonic } \\
\text { foraminifers/gram }\end{array}$ & $\begin{array}{r}\text { Sand } \\
(\%)\end{array}$ & $\begin{array}{c}\text { Mud } \\
(\%)\end{array}$ \\
\hline $45 X-2,40-42$ & 415.30 & 162.0 & 2.2 & 97.8 \\
$45 X-3,41-43$ & 416.81 & 824.4 & 2.3 & 97.7 \\
$45 X-4,38-40$ & 418.28 & 1867.7 & 10.3 & 89.7 \\
$45 X-5,40-42$ & 419.80 & 282.3 & 17.2 & 82.7 \\
$45 X-6,38-40$ & 421.80 & 708.8 & 25.0 & 75.0 \\
$45 X-7,37-39$ & 422.77 & 356.6 & 1.9 & 98.1 \\
$46 X-1,40-42$ & 423.40 & 1841.1 & 18.2 & 81.8 \\
$46 X-2,40-42$ & 424.90 & 803.0 & 10.9 & 89.1 \\
$46 X-3,40-42$ & 426.40 & 608.0 & 14.8 & 85.2 \\
$46 X-4,40-42$ & 427.90 & 563.4 & 1.5 & 98.5 \\
$46 X-5,40-42$ & 429.40 & 563.4 & 18.7 & 81.3 \\
$46 X-6,40-42$ & 430.90 & 531.6 & 21.9 & 78.1 \\
$47 X-1,40-42$ & 432.90 & 656.8 & 9.0 & 81.0 \\
$47 X-2,40-42$ & 434.40 & 375.1 & 16.9 & 83.1 \\
$47 X-3,40-42$ & 435.90 & 109.5 & 18.0 & 82.0 \\
$47 X-4,40-42$ & 437.40 & 2563.9 & 5.1 & 94.9 \\
$47 X-5,40-42$ & 438.90 & 844.5 & 5.9 & 94.1 \\
$47 X-6,40-42$ & 440.40 & 671.3 & 5.7 & 94.3 \\
$47 X-7,40-42$ & 441.90 & 457.1 & 7.5 & 92.5 \\
$48 X-1,40-42$ & 442.40 & 326.3 & 17.0 & 83.0 \\
$48 X-2,40-42$ & 443.90 & 457.8 & 6.8 & 93.2 \\
$48 X-3,40-42$ & 445.40 & 391.2 & 17.2 & 82.8 \\
$48 X-4,40-42$ & 446.90 & 731.2 & 6.3 & 93.7 \\
$48 X-5,40-42$ & 448.40 & 337.0 & 1.5 & 98.5 \\
$48 X-6,40-42$ & 449.90 & 702.9 & 3.0 & 97.0 \\
$48 X-7,38-40$ & 451.38 & 653.9 & 6.2 & 93.8 \\
$49 X-1,40-42$ & 451.90 & 837.0 & 6.2 & 93.8 \\
$49 X-3,40-42$ & 454.90 & 95.0 & 18.2 & 81.8 \\
$49 X-4,40-42$ & 456.40 & 1494.5 & 6.2 & 93.8 \\
$49 X-5,40-42$ & 457.90 & 429.8 & 6.9 & 93.1 \\
$49 X-6,40-42$ & 459.40 & 792.9 & 11.4 & 88.6 \\
$49 X-7,40-42$ & 460.40 & 644.7 & 7.4 & 92.6 \\
$50 X-1,40-42$ & 461.60 & 996.9 & 11.2 & 88.8 \\
$50 X-2,40-42$ & 463.10 & 1327.3 & 5.8 & 94.2 \\
$50 X-3,40-42$ & 464.60 & 500.6 & 2.9 & 97.1 \\
$50 X-4,40-42$ & 466.10 & 550.0 & 11.3 & 88.7 \\
$50 X-5,40-42$ & 467.60 & 1290.3 & 5.6 & 94.4 \\
$51 X-1,40-42$ & 471.30 & 1023.2 & 1.9 & 98.1 \\
$51 X-2,40-42$ & 472.80 & 418.4 & 8.1 & 91.9 \\
$51 X-3,40-42$ & 474.30 & 496.2 & 11.4 & 88.6 \\
$51 X-4,40-42$ & 475.80 & 598.9 & 5.5 & 94.5 \\
$51 X-5,40-42$ & 477.30 & 193.7 & 8.8 & 91.2 \\
$51 X-6,40-42$ & 478.80 & 505.1 & 15.1 & 84.9 \\
$51 X-7,40-42$ & 480.30 & 365.3 & 3.9 & 96.1 \\
$52 X-1,40-42$ & 480.90 & 340.7 & 4.7 & 95.3 \\
$52 X-2,40-42$ & 482.40 & 417.3 & 15.3 & 84.7 \\
$52 X-3,41-43$ & 483.91 & 273.7 & 6.4 & 93.6 \\
$52 X-4,41-43$ & 485.41 & 489.5 & 1.9 & 98.1 \\
& & & & \\
\hline
\end{tabular}

\begin{tabular}{|c|c|c|c|c|}
\hline $\begin{array}{l}\text { Core, section, } \\
\text { interval }(\mathrm{cm})\end{array}$ & $\begin{array}{l}\text { Depth } \\
\text { (mbsf) }\end{array}$ & $\begin{array}{c}\text { Planktonic } \\
\text { foraminifers/gram }\end{array}$ & $\begin{array}{l}\text { Sand } \\
(\%)\end{array}$ & $\begin{array}{c}\text { Mud } \\
(\%)\end{array}$ \\
\hline $52 X-5,42-44$ & 486.92 & 775.6 & 5.3 & 94.7 \\
\hline $52 X-6,41-43$ & 488.41 & 604.8 & 4.9 & 95.1 \\
\hline $53 X-1,39-41$ & 490.59 & 435.3 & 10.0 & 90.0 \\
\hline $53 \mathrm{X}-2,40-42$ & 492.10 & 836.3 & 8.0 & 92.0 \\
\hline $53 X-3,39-41$ & 493.59 & 422.6 & 3.2 & 96.8 \\
\hline $53 X-4,40-42$ & 495.10 & 486.4 & 24.5 & 75.5 \\
\hline $53 \times-5,39-41$ & 496.59 & 251.7 & 32.3 & 67.7 \\
\hline $53 X-6,40-42$ & 498.10 & 546.2 & 8.7 & 91.3 \\
\hline $53 \times-7,39-41$ & 499.59 & 715.9 & 12.4 & 87.6 \\
\hline $54 \mathrm{X}-1,39-41$ & 500.19 & 682.9 & 15.8 & 84.2 \\
\hline $54 \mathrm{X}-2,40-42$ & 501.70 & 345.7 & 9.6 & 90.4 \\
\hline $54 \mathrm{X}-3,39-41$ & 503.19 & 571.2 & 2.1 & 98.8 \\
\hline $54 X-4,40-42$ & 504.70 & 645.6 & 5.3 & 94.7 \\
\hline $54 X-5,40-42$ & 506.20 & 601.2 & 2.2 & 97.8 \\
\hline $54 \mathrm{X}-5,108-112$ & 506.86 & 450.9 & 7.9 & 92.1 \\
\hline $54 \mathrm{X}-6,40-42$ & 507.70 & 897.8 & 3.9 & 96.1 \\
\hline $55 \mathrm{X}-1,36-38$ & 509.86 & 462.7 & 2.6 & 97.4 \\
\hline $55 \mathrm{X}-2,40-42$ & 511.40 & 406.9 & 11.8 & 88.2 \\
\hline $55 X-3,40-42$ & 512.90 & 142.5 & 1.7 & 98.3 \\
\hline $55 X-4,40-42$ & 514.40 & 413.9 & 9.6 & 90.4 \\
\hline $55 X-5,38-40$ & 515.88 & 423.6 & 1.8 & 98.2 \\
\hline $55 X-6,38-40$ & 517.38 & 273.8 & 18.9 & 81.1 \\
\hline $55 X-7,39-41$ & 518.39 & 555.2 & 3.3 & 96.7 \\
\hline $56 \mathrm{X}-1,39-41$ & 519.19 & 777.2 & 6.8 & 93.2 \\
\hline $56 \times-2,39-41$ & 520.69 & 392.8 & 3.3 & 96.7 \\
\hline $56 \mathrm{X}-3,39-41$ & 522.19 & 432.9 & 7.0 & 93.0 \\
\hline $56 \times-4,39-41$ & 523.69 & 451.1 & 2.7 & 97.3 \\
\hline $56 \mathrm{X}-5,39-41$ & 525.19 & 409.5 & 3.7 & 96.3 \\
\hline $58 \mathrm{X}-1,39-41$ & 529.79 & 63.5 & 45.7 & 54.3 \\
\hline $58 \mathrm{X}-2,39-41$ & 531.29 & 88.1 & 52.1 & 47.9 \\
\hline $58 \times-3,39-41$ & 532.79 & 75.2 & 57.4 & 42.6 \\
\hline $58 \times-4,39-41$ & 534.29 & 1045.0 & 5.8 & 94.2 \\
\hline $58 \times-5,40-42$ & 535.80 & 267.3 & 27.4 & 72.6 \\
\hline $58 \times-6,39-41$ & 537.29 & 256.6 & 4.7 & 95.3 \\
\hline $59 \mathrm{X}-2,42-44$ & 539.92 & 453.8 & 19.8 & 80.2 \\
\hline $59 \times-3,39-41$ & 541.39 & 284.4 & 44.0 & 56.0 \\
\hline $59 \times-4,40-42$ & 542.90 & 1107.4 & 27.2 & 72.8 \\
\hline $59 \mathrm{X}-5,42-44$ & 544.42 & 94.5 & 25.7 & 74.3 \\
\hline $59 X-6,39-41$ & 545.89 & 192.2 & 4.9 & 95.1 \\
\hline $60 \times-1,39-41$ & 548.09 & 907.9 & 3.6 & 96.4 \\
\hline $61 X-1,40-42$ & 557.70 & 501.0 & 27.7 & 72.3 \\
\hline $61 X-2,40-42$ & 559.20 & 47.6 & 12.6 & 87.4 \\
\hline $61 X-3,40-42$ & 560.70 & 81.9 & 42.6 & 57.4 \\
\hline $61 \times-4,40-42$ & 562.20 & 166.1 & 37.8 & 62.2 \\
\hline $61 X-5,40-42$ & 563.70 & 31.5 & 45.8 & 54.2 \\
\hline $61 \times-6,40-42$ & 564.70 & 256.8 & 6.0 & 94.0 \\
\hline $62 X-1,18-20$ & 567.18 & 437.9 & 2.1 & 97.9 \\
\hline
\end{tabular}

Keller (1995) favored the $>106-\mu \mathrm{m}$ fraction. Martin and Liddell (1989) advocated analysis of multiple size fractions, and Kellog (1984) proposed the $>150-\mu \mathrm{m}$ fraction as a standard to avoid taxonomic problems with juvenile specimens. Although the arguments of these authors are compelling (to varying degrees depending upon the purpose of a given study), the $>150-\mu \mathrm{m}$ fraction was used here because (1) so many published accounts of planktonic foraminifers have used it, and (2) the sheer numbers of specimens in some samples precluded counting smaller size fractions. For the purposes of biostratigraphy and paleoecological reconstruction, examination of the $>63-\mu \mathrm{m}$ fraction is essential (Schroeder et al., 1987; Snyder et al., this volume). But the primary concern when comparing studies of abundance trends is that data are based on the same size fraction. Hence, results presented here are directly comparable only to other studies utilizing the $>150-\mu \mathrm{m}$ size fraction. Two-hundred sixty-four samples from Hole $904 \mathrm{~A}$ were processed and analyzed in the manner described above (Table 1).

All specimens larger than $150 \mu \mathrm{m}$ were counted in each sample split. Splits varied from the entire washed sand fraction to an aliquot representing $1 / 64$ th of that fraction. Then the raw count was multiplied by the appropriate factor to estimate total number of planktonic foraminifers in the sample. This estimated total number was then divided by grams of sediment that actually disaggregated, in order to calculate the number of specimens per gram (dry weight) of sediment (Table 1). Because the number of specimens per gram varied from 0 to 7780 , counts were plotted as $\log _{10}$ of planktonic specimens per gram (Fig. 3). Samples with one or fewer specimens per gram were plotted as zero to avoid negative values, which would add nothing to the information content of the figure.

\section{RESULTS}

In attempting to reconstruct timing and magnitude of ancient global sea-level fluctuations, changes in the abundance of planktonic specimens may help to decipher the eustatic signal. However, abundance patterns can also be influenced or controlled by a variety of local processes which can overprint the eustatic signal (Snyder and Snyder, 1993). Dramatic fluctuations in the abundance of planktonic foraminifers at Site 904 must, therefore, be interpreted with caution.

The single most dramatic change at Site 904 coincides with the change from pelagic carbonates of the Eocene through mixed carbonate and siliciclastic sediments of the Oligocene-lower Miocene to hemipelagic sediments of the middle and upper Miocene (Fig. 3). Planktonic foraminifers are consistently more abundant in carbonates (up to three orders of magnitude more abundant). This major decrease in abundance parallels changes in paleoclimate and terrigenous sediment input outlined by Poag and Ward (1993) for the U.S. middle Atlantic margin.

Superimposed on this major decline in abundance are short-term changes, usually of lesser magnitude but sometimes approaching the scale of change from Eocene to upper Miocene. Some intervals with 

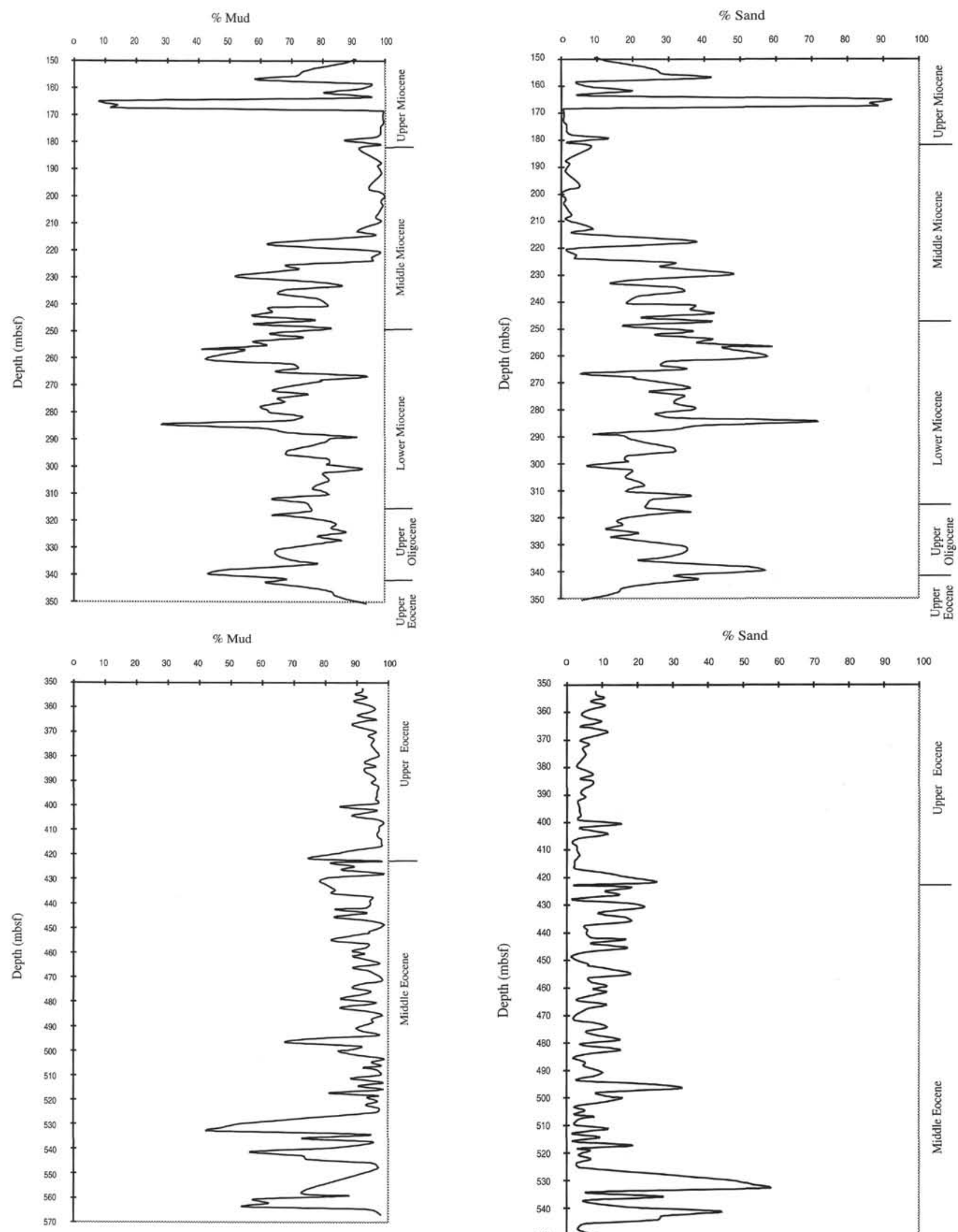

Figure 1. Percentage of mud in the Eocene-Oligocene-Miocene section of Hole 904A. Data are from Table 1.

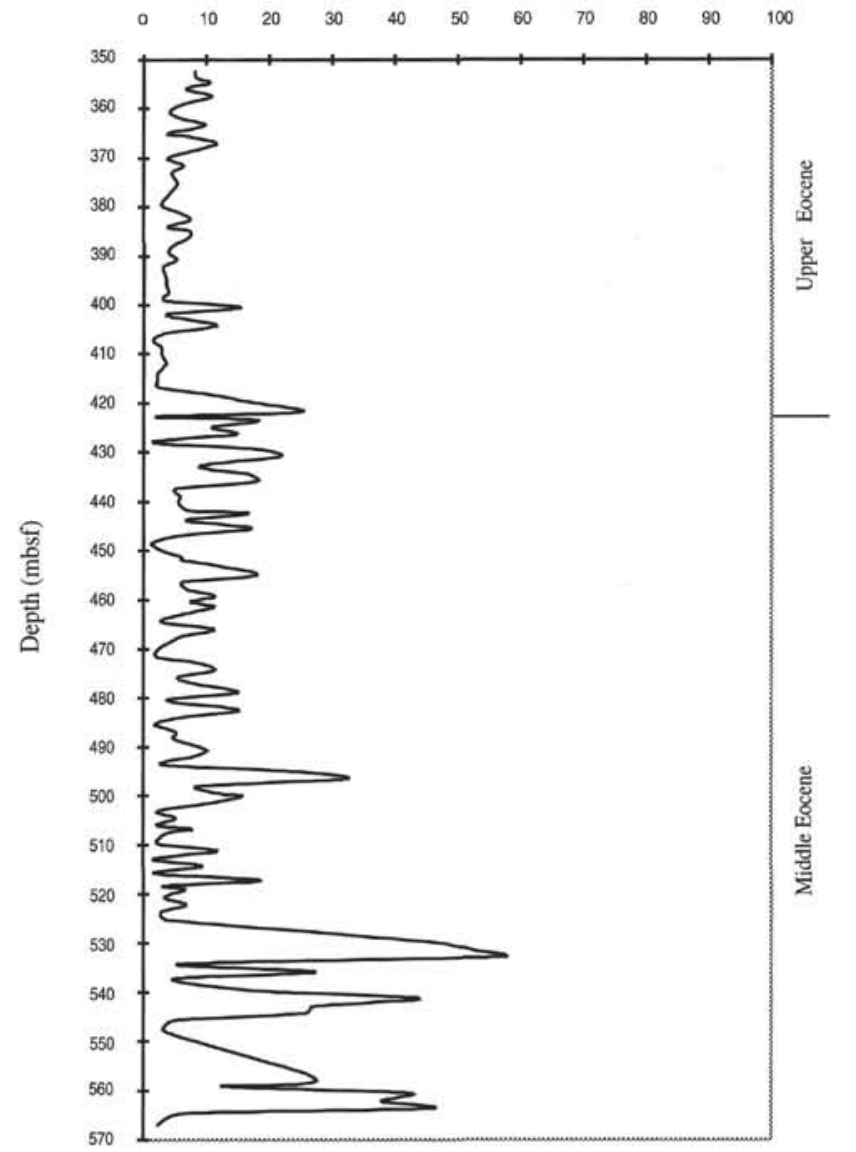

Figure 2. Percentage of sand in the Eocene-Oligocene-Miocene section of Hole 904A. Data are from Table 1. 

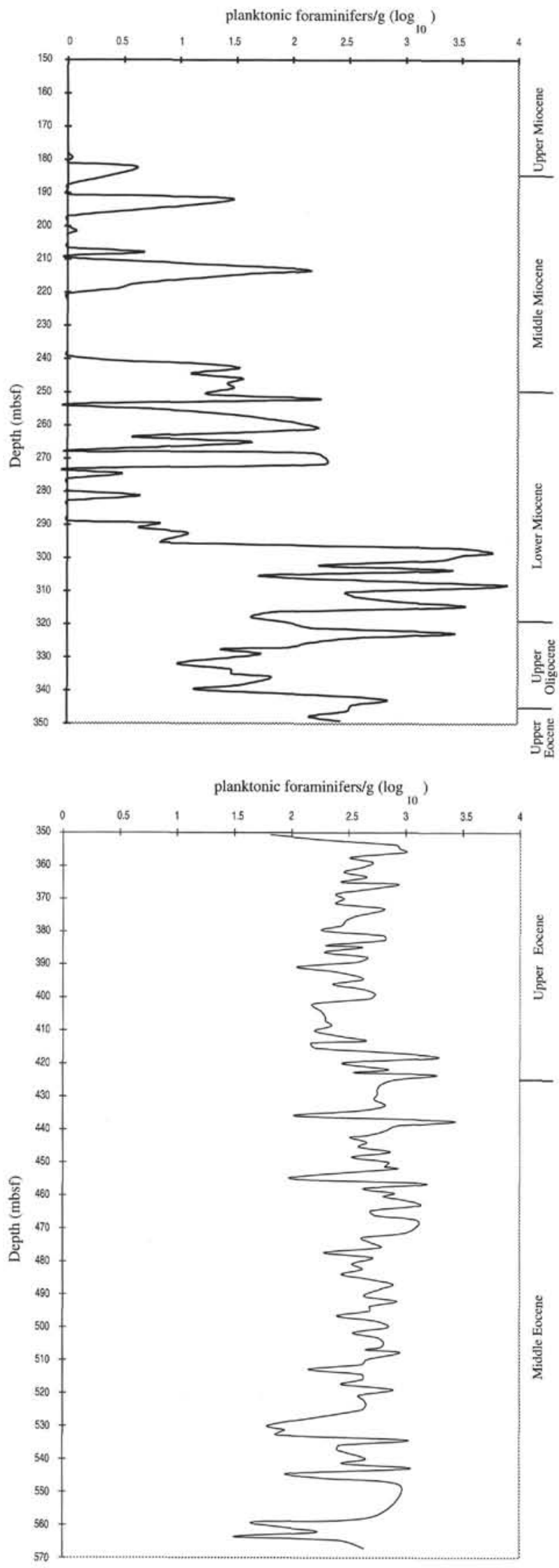

Figure 3. $\log _{10}$ of planktonic foraminifer specimens per gram of sediment in the Eocene-Oligocene-Miocene section of Hole 904A. Data are from Table 1. low abundances correspond to changes in lithology (e.g., at about 560 and $530 \mathrm{mbsf}$ at the bottom and top of a siliceous interval). Others correspond to zones of secondary mineralization, such as the pyrite cement and nodules at about $455 \mathrm{mbsf}$. The 15 -m interval above an upper Eocene tektite-bearing horizon, characterized by reworked middle Eocene specimens (Snyder et al., this volume; McHugh et al., this volume), has fewer foraminifers than sediments immediately above and below. Low abundances characterize some unconformities, such as the one at the Eocene-upper Oligocene contact at about $350 \mathrm{mbsf}$. Intervals of extremely low abundance in the OligoceneMiocene section may be related to terrigenous sediment input and/or diagenesis. Conversely, intervals of peak abundance (e.g., those at about 272,261 , and $254 \mathrm{mbsf}$ ) may reflect eustacy or decreased terrigenous sediment input, or simply may be horizons that escaped diagenetic destruction.

\section{SUMMARY}

The planktonic foraminifer abundance patterns observed at Site 904 are complex, possibly related to global sea-level changes as well as to a variety of other processes (e.g., diagenesis, paleoproductivity, local sediment accumulation rate). Deciphering this complex signal to identify the portion that reflects sea-level fluctuation will be difficult. The data in this report delineate abundance changes at Site 904 so that subsequent studies may address the possible relationship of abundance to sea-level changes along the New Jersey continental margin.

\section{ACKNOWLEDGMENTS}

Grants from the U.S. Science Support Program, funded by the National Science Foundation, supported cruise participation and postcruise research. Critical reviews by Ronald E. Martin, Richard K. Olsson, and C. Wylie Poag improved the manuscript.

\section{REFERENCES}

Haq, B.U., Hardenbol, J., and Vail, P.R., 1987. Chronology of fluctuating sea levels since the Triassic. Science, 235:1156-1167.

Kellog, T.B., 1984. Paleoclimatic significance of subpolar foraminifera in high latitude marine sediments. Can. J. Earth Sci., 21:189-193.

Loutit, T.S., Hardenbol, J., Vail, P.R., and Baum, G.R., 1988. Condensed sections: the key to age dating and correlation of continental margin sequences. In Wilgus, C.K., Hastings, B.S., Ross, C.A., Posamentier, H.W., Van Wagoner, J., and Kendall, C.G.St.C. (Eds.), Sea-Level Changes: An Integrated Approach. Spec. Publ.-Soc. Econ. Paleontol. Mineral., 42:183-213.

Lu, G., and Keller, G., 1995. Planktonic foraminiferal faunal turnovers in the subtropical Pacific during the late Paleocene to early Eocene. J. Foraminiferal Res., 25:97-116.

Martin, R.E., and Liddell, W.D., 1989. Relation of counting methods to taphonomic gradients and biofacies zonation of foraminiferal sediment assemblages. Mar. Micropaleontology, 15:67-89.

Miller, K.G., Mountain, G.S., and Shipboard Scientific Party, 1994. Introduction. In Mountain, G.S., Miller, K.G., Blum, P., et al., Proc. ODP, Init. Repts., 150: College Station, TX (Ocean Drilling Program), 5-9.

Poag, C.W., and Ward, L.W., 1993. Allostratigraphy of the U.S. middle Atlantic continental margin-characteristics, distribution, and depositional history of principal unconformity-bounded Upper Cretaceous and Cenozoic sedimentary units. Geol. Surv. Prof. Pap. U.S., 1542:1-81.

Schroeder, C.J., Scott, D.B., and Medioli, F.S., 1987. Can smaller benthic foraminifera be ignored in paleoenvironmental analyses? J. Foraminiferal Res., 17:101-105.

Snyder, S.W., and Snyder, S.W., 1993. Biotic response in the sequence stratigraphic framework: overprint of local conditions vs. eustacy. In Armentrout, J. (Ed.), Rates of Geologic Processes. Gulf Coast Sect., Soc. Econ. Paleontol. Mineral., Ann. Res. Conf., 14:235-242.

Van Wagoner, J.C., Posamentier, H.W., Mitchum, R.M., Jr., Vail, P.R., Sarg, J.F., Loutit, T.S., and Hardenbol, J., 1988. An overview of the fundamen- 
tals of sequence stratigraphy and key definitions. In Wilgus, C.K., Hast-

ings, B.S., Ross, C.A., Posamentier, H.W., Van Wagoner, J., and

Kendall, C.G.St.C. (Eds.), Sea-Level Changes: An Integrated Approach. Spec. Publ.-Soc. Econ. Paleontol. Mineral., 42:39-45.
Date of initial receipt: 28 February 1995

Date of acceptance: 26 July 1995

Ms 150SR-002 